\title{
Procesos de reproducción social e integración sociolaboral. Trayectorias de inserción al mercado de trabajo en el Conurbano bonaerense*
}

\author{
Processes of social reproducition and laboral integration. Labor market \\ insertion trajectories in Buenos Aires suburbs
}

Guillermina Comas*

Juan Ignacio Bonfiglio ${ }^{* * *}$

\section{Resumen}

El artículo tiene por objetivo analizar las transformaciones en los procesos de reproducción social a partir de los recorridos que caracterizaron la inserción de la fuerza laboral al mercado de trabajo y de los sentidos que los trabajadores atribuyen a su vida laboral en el presente.

La investigación se realizó a partir del análisis de las trayectorias y los relatos de trabajadores residentes en un espacio territorialmente segregado y con altos niveles de informalidad laboral situado en la periferia del Conurbano bonaerense. El diseño metodológico combinó una fase cuantitativa, en la que se abordan los procesos de inserción a partir de las trayectorias laborales y un análisis cualitativo, orientado a reconstruir los sentidos que estos trabajadores le asignan a sus acciones en el

\footnotetext{
* Artículo recibido el 11 de Abril de 2016. Aceptado el 14 de Junio de 2016.

** Doctora en Ciencias Sociales. Pertenencia Institucional: Instituto de Investigaciones Gino Germani. Facultad de Ciencias Sociales. UBA. E-mail: comasgui@gmail.com

**** Licenciado en Sociología. Pertenencia Institucional: Observatorio de la Deuda Social Argentina (ODSAUCA). E-mail: jbonfiglio@gmail.com
} 
De Prácticas y discursos/ Universidad Nacional del Nordeste/ Centro de Estudios Sociales

plano laboral. Los datos fueron relevados por una encuesta que asumió el formato de calendario de eventos diseñado para captar aspectos de los recorridos laborales y vitales de los encuestados y un relevamiento cualitativo, realizado en el 2014, a partir de entrevistas en profundidad a una submuestra de casos encuestados anteriormente.

Los datos muestran una disminución en las chances de movilidad en las trayectorias de ingreso al mercado y un endurecimiento del origen social sobre las posibilidades de logro de un recorrido laboral de calidad. No obstante, estos comportamientos no se traducen de manera directa en el plano de las representaciones y expectativas subjetivas.

\section{Palabras clave}

Trayectorias laborales -reproducción social- metodología mixta

\section{Abstract}

The objective of this article is to analyze the changes in the processes of social reproduction from changes in the paths that characterized the entry in the labor market and senses that workers build in relation to their working lives.

This work will carry out an analysis of the trajectories of insertion into the labor market and the meanings attributed to the work of resident workers in territorially segregated neighbor with high levels of informality located on the Buenos Aires suburbs. The research was conducted from a design that combines a quantitative phase in which the processes of integration into the labor market are analyzed from the career paths, the data were collected by a survey that took calendar format work events specially designed to capture aspects of labor and life trajectories of respondents and a qualitative survey conducted in 2014 from in-depth interviews with a subsample of cases that had been surveyed previously. 
The data show, a decrease in the chances of mobility trajectories entering the market and a tightening of social origin on the possibilities of quality employment path. However, this does not necessarily translate into the domain of representations and subjective expectations regarding their employment situation and the chances of labor mobility.

\section{Keywords}

Labor trajectory-social reproduction-mixed methodology.

\section{Introducción}

El artículo se propone dar cuenta de los ejes de diferenciación al interior de los sectores populares a partir de un análisis de las trayectorias laborales, consideradas como expresión de las posibilidades de inserción al mercado de trabajo. Al analizar las trayectorias de ingreso y los relatos de un grupo de trabajadores que residen en una localidad periférica del Conurbano bonaerense, proponemos una aproximación a los procesos de reproducción social asociados al trabajo.

El mercado de trabajo constituye una dimensión central para abordar las transformaciones que experimentó la sociedad argentina de las últimas cuatro décadast. Diferentes estudios sostienen la emergencia de una nueva matriz de marginalidad que comienza a configurarse en la década del setenta y que se consolida durante los años noventa, persistiendo en las primeras décadas del siglo XXI, a pesar de las visibles mejoras respecto a la crisis de la convertibilidad. Si consideramos que, en el marco de esta nueva matriz de marginalidad, se evidencian transformaciones en los

\footnotetext{
${ }^{\dagger}$ Tras las reformas estructurales que se inician a mediados de los setenta con las políticas de apertura y ajuste fiscal y se consolidarían en los años noventa, se desencadenó un proceso de deterioro de los mercados de trabajo que se manifestó en un notable incremento de la precarización del empleo asalariado y de la informalidad laboral. Tras la crisis y caída del modelo aperturista, se desarrolló una dinámica política-económica donde el Estado volvió a asumir un papel relevante en la regulación de las actividades económicas y, principalmente, en el estímulo del mercado interno con un fuerte incremento de la actividad económica, acompañado de una recuperación del consumo y del empleo. Distintos estudios coinciden en señalar que el grueso de los empleos creados en esta etapa, contrariamente a lo que había sucedido en la última mitad de la década del noventa, habían sido empleos registrados en el marco de la recuperación de los convenios colectivos de trabajo (Neffa y Panigo, 2009). Sin embargo, estos elementos coexisten con evidencias que permitirían sostener la hipótesis de la persistencia de la fragmentación laboral, cuya más clara manifestación la constituye la imposibilidad del mercado de trabajo formal para absorber a la totalidad de la fuerza de trabajo (Salvia, Vera y Poy, 2015).
} 
De Prácticas y discursos/ Universidad Nacional del Nordeste/ Centro de Estudios Sociales

patrones que producen y a partir de los cuales se reproducen las situaciones de marginalidad, cabe analizar los cambios que tienen lugar en los procesos de reproducción intergeneracional e intrageneracional, los que mostrarían menos chances de movilidad ocupacional para las nuevas generaciones y una serie de mutaciones respecto de los significados relativos al trabajo.

Durante décadas el ingreso al mundo laboral constituyó un pasaje no problemático: la familia, el sistema educativo y el mercado laboral establecían los mecanismos que regulaban el tránsito entre estos espacios. Las transformaciones estructurales alteraron ese proceso volviéndolo, especialmente para los jóvenes de sectores populares, crecientemente precario y más vinculado a la reproducción de las condiciones de origen (Jacinto, 2010; Pérez, 2008; Salvia y otros, 2008). Podría sostenerse, que los cambios en las formas en las que la población activa se integra al mundo laboral constituyen expresión de las transformaciones en los mecanismos de integración social y de reproducción de los factores que definen la inclusión/exclusión al mercado de trabajo formal.

En este trabajo nos proponemos analizar las transformaciones en los procesos de reproducción social a partir de los cambios en los recorridos que caracterizaron la inserción de la fuerza de trabajo al mercado y de los sentidos construidos por los trabajadores en relación a su vida laboral, interpretándolos en clave de las segmentaciones que caracterizan a la estructura del mercado laboral. En este sentido, se apunta a indagar sobre la existencia de procesos de precarización y heterogeneización de las inserciones laborales. En este marco, los mecanismos de movilidad hacia las posiciones más integradas resultan cada vez más difíciles y el despliegue de estrategias tradicionales, por parte de la fuerza de trabajo, no resulta suficiente.

Los procesos de precarización son caracterizados desde dos dimensiones: en primer lugar, analizando de manera cuantitativa las "trayectorias de inserción al mercado de trabajo". Cabe afirmar que las transiciones ocupacionales que tienen lugar durante el proceso de inserción definen una tendencia respecto a la lógica de las trayectorias en el largo plazo, por lo cual su análisis resulta una estrategia interesante para dar cuenta de 
De Prácticas y discursos/ Universidad Nacional del Nordeste/ Centro de Estudios Sociales

las transformaciones dentro de patrones de reproducción más amplios (García Blanco y Gutiérrez, 1996; Pérez Islas y Urteaga, 2001). En segundo lugar, planteamos una aproximación interpretativa con la intención de captar, a partir de la experiencia vital de estos trabajadores, los vínculos entre estos procesos y sus historias personales (Graffigna, 2005). Mediante la complementación de ambas perspectivas metodológicas, intentamos reconstruir las articulaciones y disociaciones entre los mecanismos de cambio social en la estructura socio-laboral- (vistos aquí a través de los mecanismos de segmentación en las trayectorias de inserción) y los sentidos que los trabajadores le asignan a sus acciones en el plano laboral, mediante valoraciones, decisiones y estrategias.

\section{Los enfoques de trayectorias: supuestos teóricos y analíticos de estas} perspectivas en los estudios de jóvenes

La contribución de la mirada longitudinal en los estudios laborales ha sido extensamente tratada en la literatura académicał. Si bien en la Argentina aún existen importantes limitaciones - particularmente para los estudios de tipo cuantitativo-, en la última década han proliferado investigaciones que, desde distintas perspectivas teóricas y abordajes metodológicos, tuvieron a las trayectorias laborales como eje en el estudio de distintos procesos asociados al mundo del trabajo (Muñiz Terra, 2008; Jacinto, 2010; Panaia, 2006; Salvia y Chávez Molina, 2002; Salvia, Persia y De Grande, 2000). Cabe destacar además que constituyó una perspectiva fértil para los estudios de jóvenes (Saraví, 2006; Otero, 2009; Roberti, 2014; Jacinto y Chitarroni, 2010; Pérez, Deleo y Fernández Massi, 2014; Molina Derteano, 2007, entre otros).

El proceso de inserción al mercado de trabajo es la etapa de la trayectoria ocupacional en la que se inician las carreras laborales, allí un conjunto de factores asociados a los capitales familiares, individuales, así como a aspectos institucionales, inciden en las posibilidades de incorporación en dinámicas caracterizadas por la

\footnotetext{
* Los distintos aportes se desprenden principalmente de los desarrollos teóricos ligados al enfoque biográfico (Bertaux, 1999) y a las diversas vertientes del enfoque biografía-sociedad (Elder, 1998; Pries, 1993 y 1996). Las distintas perspectivas constituyen formas alternativas de dar cuenta de la articulación entre las dinámicas individuales y la estructura social desde una perspectiva longitudinal como herramienta para el estudio de los procesos sociales.
} 
De Prácticas y discursos/ Universidad Nacional del Nordeste/ Centro de Estudios Sociales

acumulación de experiencia y trayectorias ascendentes o bien en recorridos estancados en posiciones precarias e inestables (Saraví, 2006;García Blanco y Gutiérrez, 1996). Además, los cambios más importantes en las posiciones de las carreras laborales tienen lugar en las etapas iniciales de la vida activa, por lo tanto los eventos ocupacionales que tienen lugar durante el proceso de inserción definen en buena parte la lógica que asumirán las trayectorias en el largo plazo (Parrado, 2005; Pérez Islas y Urteaga, 2001).

En este marco se considera que: la dinámica de ingreso al mercado de trabajo, la cercanía (social y temporal) respecto a la posición social de origen, así como las tendencias generales que se observen en términos de integración sociolaboral, pueden dar cuenta de los procesos de cambio social. Los estudios sobre transiciones han destacado cómo estos procesos de tránsito constituyen una etapa de "cristalización de las desigualdades sociales". Eventos vitales como: la salida de la escuela, la entrada al mundo del trabajo, la salida del hogar de origen, entre otros, desencadenan procesos, en tanto influyen en la configuración de acontecimientos futuros en la biografía de los jóvenes (Casal, 2001; Mora Salas y Oliveira, 2014). Los enfoques centrados en la perspectiva de la acumulación de desventajas se nutren teórica y metodológicamente de este tipo de análisis (Saravi, 2006; De la Rocha, 2004). En este sentido, los itinerarios biográficos que tienen lugar en lo que se conoce como el proceso de transición a la adultez están atravesados por experiencias de vida en las que los jóvenes toman decisiones en contextos de opciones más o menos restringidas (Minor Salas y Oliveira, 2014).

Dadas las características regresivas que asumió el proceso de cambio social en la Argentina durante el último cuarto del siglo XX, cabría esperar que esta dinámica haya tenido impacto sobre el ingreso a la vida laboral de la población de estudio, por una parte, precarizando sus trayectorias de inserción y, por otra, modificando el efecto del origen social sobre las características que asumieron las mismas. A su vez, sostenemos que, desde una mirada retrospectiva, los significados configurados en torno el trabajo representarían estos procesos marcando diferencias entre los diferentes tipos de origen social y trayectorias. 


\section{Principales coordenadas metodológicas del estudio}

La investigación que da origen a este artículo es resultado de dos proyectos que abordaron de manera continua un estudio de caso mediante una estrategia metodológica mixta. La etapa de diseño cuantitativo requirió la elaboración de un esquema causal y de la operacionalización de las variables de estudio sobre los insumos proporcionados por una encuesta propia, diseñada para captar las particularidades de las trayectorias laborales y familiares de los entrevistados§.

El diseño cuantitativo respondió a una hipótesis de trabajo orientada a dar cuenta de los cambios en los procesos de inserción al mercado de trabajo de dos generaciones de trabajadores que residían en un espacio territorial con altos niveles de informalidad laboral y segregación residencial. Se planteó un análisis comparativo, teniendo en cuenta el peso del origen social sobre las posibilidades de desarrollar trayectorias de integración. En este sentido, se buscó contrastar empíricamente en qué medida las transformaciones regresivas, en el marco de las cuales las generaciones jóvenes tendieron a transitar su entrada al mundo laboral, impactaron sobre las trayectorias laborales que desarrollaron hasta una edad intermedia del ciclo vital.

Las variables a definir en términos operativos fueron: la trayectoria de inserción al mercado de trabajo, la cohorte de ingreso al mercado de trabajo y el origen social. La trayectoria de inserción se definió temporalmente tomando el primer empleo como punto de partida y como punto de llegada el empleo a los 35 años. La selección del primer empleo como punto de inicio de la trayectoria, respondió a la necesidad de fijar el punto de ingreso a la vida económicamente activa mientras que, la determinación de la edad de 35 años como momento en el que finaliza la etapa de inserción al mercado de trabajo, se apoya sobre la noción de "madurez ocupacional" (Erikson y Goldthorpe, 1993). Este sería el punto en el que el sujeto ya habría recorrido una parte importante de su carrera ocupacional, pudiendo por lo tanto definirse una tendencia más o menos clara respecto a su evolución general.

\footnotetext{
${ }^{\S}$ La encuesta se realizó en el año 2008 en la localidad de Ministro Rivadavia, partido de Almirante Brown, en el Conurbano bonaerense. El diseño de la encuesta combinó un formato tradicional de encuesta de hogares con un calendario de eventos laborales y familiares concebido para captar distintos aspectos de la trayectoria vital del respondente. El diseño muestral fue no probabilístico y relevó 560 casos en una muestra por cuotas de sexo, edad y categoría ocupacional extraídas de parámetros censales.
} 
De Prácticas y discursos/ Universidad Nacional del Nordeste/ Centro de Estudios Sociales

En función de las posiciones iniciales y las posiciones alcanzadas se construyeron tres categorías de trayectorias: trayectorias no precarias, identifica a los casos que siempre se mantuvieron en el segmento primario; trayectorias ascendentes, que pasan del segmento secundario al primario, y trayectorias precarias, que son los casos que se mantuvieron desde el primer empleo en ocupaciones del segmento secundario o descendieron.

Por otra parte, se definieron dos criterios de estratificación: uno temporal, identificado a partir de la cohorte según el año del primer empleo**, y otro definido desde el origen social que toma como criterios centrales las características de la ocupación del principal aportante de ingresos del hogar cuando el entrevistado tenía 14 años (Ver Tabla de definición en el Anexo).

En la segunda fase se desarrolló un diseño cualitativo, con el objetivo de indagar, ya en el plano del mundo de las significaciones (Kornblit, 2004), los sentidos atribuidos por estos trabajadores a su experiencia de vida en el plano laboral. Cabe aclarar que no nos interesó tanto una reconstrucción cronológica de su trayectoria, sino el modo en que los entrevistados representan su situación laboral en vinculación con los eventos pasados y con los mecanismos de movilidad proyectados respecto a su futuro laboral o el de sus hijos.

En esa sección analizamos un conjunto de entrevistas semi estructuradas realizadas entre 2013 y 2014 a trabajadores jefes de hogar pertenecientes a la segunda cohorte, seleccionados de la base de datos original de manera intencional, siguiendo el modelo analítico de trayectorias de ingreso al mercado laboral elaborado en el estudio cuantitativo (ver cuadro de entrevistados en Anexo).

\footnotetext{
** Si bien tradicionalmente se tiende tomarse en cuenta la cohorte de nacimiento, la consideración de otro tipo de eventos se constituiría como un factor relevante de acuerdo a los objetivos de investigación particulares (Ryder, 1965). En este sentido, considerando la relevancia que plantean para las trayectorias ocupacionales las posiciones iniciales, definimos las cohortes en concordancia al momento de ingreso al mercado de trabajo. Definir la pertenencia a la cohorte a partir de la edad del primer empleo responde a que la segmentación del mercado de trabajo se reproduce dificultando la movilidad entre distintos segmentos. De esta manera, el ingreso en un período caracterizado por condiciones de mayor segmentación tendría como consecuencia menos probabilidades de movilidad ascendente en términos de segmentos del mercado de trabajo en el caso de que exista relación entre el cambio histórico y las trayectorias de los sujetos analizados (Piore, 1975).
} 
De Prácticas y discursos/ Universidad Nacional del Nordeste/ Centro de Estudios Sociales

Cabe aclarar que no abordaremos las representaciones de trabajadores jóvenes, sino de trabajadores que, habiendo alcanzado la edad media de la vida activa, tuvieron diferentes conductos de ingreso al mercado de trabajo. Esos circuitos, situados en la juventud de los entrevistados, suponen un proceso de entrada al trabajo que, por lo general, tuvo lugar en simultáneo a la salida temprana del sistema educativo.

\section{Trayectorias de inserción al mercado de trabajo de dos cohortes de trabajadores}

Una cohorte se define como un grupo de sujetos que experimenta un mismo evento bajo las mismas circunstancias históricas, esto implica que sus miembros comparten un conjunto de condiciones estructurales y de experiencias vitales comunes (Ryder, 1965). En este marco, el análisis de los procesos de inserción laboral de dos generaciones permite dar cuenta de las transformaciones que tuvieron lugar entre una generación y otra.

Para una población con las características de la estudiada, cabe plantear como hipótesis que la cohorte que ha desarrollado su trayectoria de inserción al mercado en el contexto de las políticas aperturistas y de ajuste estructural iniciadas a mediados de la década del setenta, evidencie, en relación a la cohorte de ingreso más antigua, una mayor proporción de trayectorias precarias, manifestando la existencia de mayores dificultades, dentro de las cuales también tendría lugar un mayor nivel de transmisión intergeneracional de esta situación. En otras palabras, cabría esperar que el origen social pese más en la cohorte más joven, limitando más las posibilidades de los trabajadores de origen más bajo.

\subsection{Características de las trayectorias de inserción para diferentes generaciones}

Si sostenemos que los procesos de inserción al mercado de trabajo pueden concluir o no con la integración al mercado de trabajo formal, resulta de interés definir qué entendemos, en términos conceptuales, por integración socio-laboral. Al retomar la perspectiva de la marginalidad económica (Nun, 1969; Quijano, 1971), reconocemos la existencia de una porción de la población activa que no es absorbida por el aparato 
productivo de los procesos dinámicos de acumulación de capital, en este sentido las condiciones que asume el proceso de acumulación contribuyen a generar una población que tiende a desarrollar actividades laborales de subsistencia.

Desde las teorías de la segmentación del mercado de trabajo se sostiene que la desigualdad, tanto en el acceso al mercado de trabajo, como al interior del mismo, no están asociadas con atributos específicos de la fuerza laboral, sino con los mecanismos institucionales que lo regulan. Siguiendo este argumento, desde la vertiente dualista (Piore, 1975), se destaca la existencia de dos mercados de trabajo: el secundario, con bajos salarios, calificaciones, estabilidad y condiciones de trabajo precarias y el primario, caracterizado por altos salarios, buenas condiciones laborales y progreso a lo largo de la carrera laboralt+.

Situados en estos enfoques, partimos del supuesto que el deterioro de las condiciones del mercado laboral tiene entre sus consecuencias un incremento en los excedentes relativos de población asociados a los procesos de marginalidad económica, esto se manifestaría en un mayor nivel de segmentación de los mercados de trabajo y en el consecuente incremento de trayectorias precarias.

Al indagar sobre la forma que asume esta asociación, observamos que los datos se comportan en el sentido de la hipótesis planteada, es decir que las trayectorias de movilidad del segmento secundario al primario representan un 43\% de la población de la primera cohorte, mientras que solo alcanzan a un $26 \%$ para la segunda. De esta manera, se nota un fuerte descenso de las trayectorias ascendentes en el marco de un contexto de apertura (ver cuadro 1 ).

\footnotetext{
${ }^{+H}$ El mercado de trabajo primario estaría vinculado a los llamados mercados internos de trabajo, las condiciones de estos no estarían regidas por la competencia, sino que las distintas instituciones regulan los procesos de reclutamiento, capacitación y promoción de la fuerza de trabajo. Los mercados de trabajo secundarios, en cambio, serían mercados competitivos en los que la oferta y la demanda definen salarios y condiciones de trabajo. Las grandes empresas en términos de productividad y control sobre el mercado son las que tienen mercados internos de trabajo, mientras que los mercados de trabajos externos o competitivos estarían asociados con los pequeños establecimientos.
} 
De Prácticas y discursos/ Universidad Nacional del Nordeste/ Centro de Estudios Sociales

Cuadro 1. Trayectorias de inserción al mercado de trabajo por cohorte de ingreso al mercado de trabajo.

\begin{tabular}{|l|l|l|}
\hline & $\begin{array}{c}\text { Ingreso al mercado de } \\
\text { trabajo antes de } 1976\end{array}$ & $\begin{array}{c}\text { Ingreso al mercado de trabajo } \\
\text { desde de } 1976\end{array}$ \\
\hline Trayectorias no precarias & $6,4 \%$ & $16,9 \%$ \\
\hline Trayectorias ascendentes & $43,4 \%$ & $25,8 \%$ \\
\hline Trayectorias precarias & $50,2 \%$ & $57,2 \%$ \\
\hline \multirow{2}{*}{ Total } & 235 & 236 \\
\cline { 2 - 4 } & $100 \%$ & $100 \%$ \\
\hline
\end{tabular}

Elaboración propia en base a relevamiento PCEyDS. Foncyt 33737

Sin embargo, junto con este "deterioro" coexiste un incremento de las trayectorias que han podido estabilizarse en posiciones laborales favorables entre la cohorte más joven $(6,4 \%$ vs $16,9 \%)$, cuya proporción más que duplica a la de la primera cohorte. Es decir que, tras los cambios estructurales, se incrementaría la proporción de trayectorias que se inician en posiciones del mercado de trabajo primario, las cuales, probablemente, al desarrollar trayectorias que desde el inicio han transcurrido en este segmento, hayan presentado mayores posibilidades de progreso en la carrera laboral.

Finalmente, en los dos períodos aumenta la proporción de trayectorias precarias caracterizadas por la permanencia o caída en el segmento secundario que pasan de un $50 \%$ a un $57 \%$. No obstante, cabe destacar que la población analizada presenta una amplia proporción de trayectorias de exclusión laboral de manera previa al proceso de apertura y reestructuración económica. En este sentido, podemos señalar que el efecto regresivo observable en las trayectorias estudiadas responde a la polarización que tiene lugar como consecuencia de la disminución de las trayectorias ascendentes.

Ahora bien, en términos de la evolución del mercado de trabajo en general, cabría esperar que en un deterioro de las condiciones generales del mercado de trabajo, la disminución de recorridos ascendentes se vea acompañada por un incremento de las trayectorias más precarias, ligadas a la permanencia en posiciones del sector secundario o a caídas en el mismo.

En este caso sucede que la disminución de trayectorias ascendentes se da al mismo tiempo que se incrementan las trayectorias estancadas en posiciones marginales, pero también a la par del aumento de los recorridos en trayectorias no precarias, las cuales 
De Prácticas y discursos/ Universidad Nacional del Nordeste/ Centro de Estudios Sociales

presentan, en términos relativos, un fuerte incremento. Este fenómeno puede interpretarse a partir de la presencia de una dinámica de polarización, donde el incremento de posiciones caracterizadas por la permanencia en un segmento específico implica menor proporción de movimientos ascendentes, evidenciando la presencia de un proceso en el que la reproducción de las condiciones de inicio de la vida laboral se vuelve más fuerte.

\subsection{Origen social y trayectorias}

El mercado de trabajo se constituye como un conjunto de relaciones socialmente estructuradas que se presentan a los individuos como marcos de opciones posibles. Estas opciones pueden conllevar más o menos oportunidades en función de la desigual distribución de distintos tipos de capital, siempre en el marco de los atributos específicos del sistema de estratificación que estén vigentes en un momento dado (Bourdieu, 1988; Pzeworski, 1982). En este sentido, es clave el papel de la herencia de oportunidades asociada a la transmisión de capital físico, cultural o social. El origen social se constituye como elemento central para dar cuenta de cómo se transmiten las ventajas o desventajas de una generación a otra; diversos estudios sobre las trayectorias juveniles toman este elemento para dar cuenta de la precariedad e inestabilidad de las trayectorias de los jóvenes según su origen social (Roberti, 2014; Pérez, Deleo y Fernández Massi, 2014; Busso, Longo y Pérez, 2014).

Al observar la distribución de los tipos de trayectoria según el estrato social de origen de los encuestados y el momento histórico de ingreso al mercado de trabajo (cuadro 2), se destaca que el proceso de polarización, observado en las relaciones anteriores, se especifica de tal manera que las trayectorias que transcurren en el segmento primario más que se duplican entre cohortes para los trabajadores de estrato medio o bajo integrado, pero también se incrementan para los de origen marginal. Sin embargo, para la segunda cohorte las probabilidades que tienen frente a los que provienen de estratos medios son 5 veces menores. Al mismo tiempo, se presenta un fuerte incremento de las trayectorias más precarias que marcan situaciones de reproducción 
De Prácticas y discursos/ Universidad Nacional del Nordeste/ Centro de Estudios Sociales

de posiciones en estratos marginales; esta dinámica tiene lugar con mayor intensidad para los trabajadores con origen social más precario.

Cuadro 2. Trayectoria de inserción al mercado de trabajo por origen social según cohorte de ingreso al mercado de trabajo

\begin{tabular}{|c|l|l|l|}
\hline \multicolumn{2}{|c|}{} & \multicolumn{1}{|l|}{$\begin{array}{c}\text { Ingreso al mercado } \\
\text { de trabajo antes de } \\
1976\end{array}$} & $\begin{array}{c}\text { Ingreso } \\
\text { mercado de trabajo } \\
\text { desde } 1976\end{array}$ \\
\hline \multirow{2}{*}{$\begin{array}{l}\text { Trayectorias no } \\
\text { precarias }\end{array}$} & Estrato marginal & $0,7 \%$ & $6,3 \%$ \\
\cline { 2 - 4 } & Estrato medio/bajo integrado & $14,0 \%$ & $30,0 \%$ \\
\hline \multirow{2}{*}{$\begin{array}{l}\text { Trayectorias } \\
\text { ascendentes }\end{array}$} & Estrato marginal & $40,7 \%$ & $23,8 \%$ \\
\hline \multirow{2}{*}{$\begin{array}{c}\text { Trayectorias } \\
\text { precarias }\end{array}$} & Estrato medio/bajo integrado & $48,4 \%$ & $27,3 \%$ \\
\cline { 2 - 4 } & Estrato marginal & $58,6 \%$ & $69,8 \%$ \\
\hline
\end{tabular}

Elaboración propia en base a relevamiento PCEyDS. Foncyt 33737

Los datos confirman que las formas que tomaron los procesos de inserción al mercado de trabajo estuvieron afectadas por las condiciones estructurales. Quienes experimentaron el proceso de transición hacia el mercado laboral en el período de apertura y ajuste estructural tuvieron más chances de experimentar procesos de inserción más precarios. Al mismo tiempo que los trabajadores de origen social próximos a estratos marginales tendieron a alejarse cada vez más de los que provenían de estratos más consolidados, a pesar que una porción de estos también haya visto empeorar su situación respecto a la cohorte precedente.

En síntesis, desde los datos presentados se observa un proceso regresivo que se manifiesta en menores chances de tener trayectorias asociadas al segmento primario del mercado de trabajo para la cohorte más joven. Al mismo tiempo, se aprecia una mayor incidencia del estrato social de origen sobre las chances de integración al empleo formal también para los más jóvenes. Estos fenómenos estarían dando cuenta de una transformación en función de mayores niveles de reproducción, tanto de las condiciones iniciales de las trayectorias de inserción al mercado de trabajo generalmente precarias- como también del estrato social de origen. En otras palabras, 
De Prácticas y discursos/ Universidad Nacional del Nordeste/ Centro de Estudios Sociales

la cohorte más joven, que inicia su historia laboral en el marco de procesos regresivos, registra mayores niveles de trayectorias de exclusión del mercado de trabajo formal con altos niveles de reproducción de estas situaciones. En este punto, cabría preguntarse si este proceso de polarización tiene un correlato a nivel simbólico, es decir si las representaciones sobre el trabajo y las posibilidades de movilidad tienen patrones homogéneos con relación a las trayectorias de inserción al mercado de trabajo y a los orígenes sociales.

\section{Una mirada interpretativa de las trayectorias laborales}

Este análisis se realizó a partir de un conjunto de entrevistas realizadas a trabajadores pertenecientes a la segunda cohorte de ingreso al mercado laboral. Los datos cuantitativos mostraron que la segmentación es más contundente para la segunda generación, lo que hace que las chances de movilidad sean reducidas y que las condiciones de inicio sean más determinantes que en otros momentos. En este sentido, nos interesó indagar sobre las construcciones subjetivas de este grupo en relación a su vida laboral a los fines de identificar, en el plano de las significaciones, la existencia de los procesos que en el apartado anterior hemos identificado en términos de chances de inserción más precarias y endurecimiento del origen social sobre las posibilidades de calidad del recorrido laboral.

Indagar sobre las trayectorias a partir de los relatos implica una aproximación no estructurada a las historias laborales. Este enfoque permite comprender de qué forma los sujetos construyen los sentidos sobre su vida laboral y cómo lo hacen en correspondencia a su contexto material y su tiempo histórico. Situados en esta perspectiva retomamos los significados en torno al trabajo, reconstruyendo enlaces o rupturas entre los mismos. A su vez, consideramos que la organización de diversas estrategias puede constituirse en un elemento capaz de reforzar o relativizar los cursos de segmentación identificados en el análisis cuantitativo.

De esta manera, el registro cualitativo evidencia rasgos perceptivos, motivacionales y actitudinales que, a través de las enunciaciones de los trabajadores (Sautu, 2011), nos permiten acceder a los procesos de segmentación laboral, pero a partir de la 
De Prácticas y discursos/ Universidad Nacional del Nordeste/ Centro de Estudios Sociales

significación que los sujetos les atribuyen. Por lo tanto, la propuesta de esta sección no apunta a replicar los patrones observados a través de la encuesta, sino a la formulación de inferencias interpretativas que consideran a esos patrones únicamente como punto de partida.

-En primer lugar, realizamos una descripción de las trayectorias según el estrato social de origen y tipo de trayectoria de ingreso, con el objeto de aproximarnos al plano de las condiciones materiales de existencia de los entrevistados. Esas descripciones están sintetizadas en la tabla $N^{\circ} 1$.

-En segundo lugar, abordamos las lecturas que los trabajadores realizan sobre su situación laboral en relación con las acciones que despliegan y a sus expectativas de movilidad socio-laboral.

\subsection{Descripción de las trayectorias por origen social: análisis empírico como aproximación a las condiciones materiales de existencia}

Este apartado constituye una descripción a partir de una combinación entre datos obtenidos en el relevamiento de la encuesta y las entrevistas en profundidad. Los relatos de vida se apoyan sobre la realidad histórico empírica, articulando el contexto histórico y el tiempo biográfico. Por eso, describimos los principales hechos que caracterizan las trayectorias de los entrevistados y que constituyen una aproximación al conocimiento de sus condiciones materiales de existencia, teniendo en cuenta su origen social y el tipo de recorrido que experimentaron en el ingreso a su vida laboral.

Al analizar los recorridos laborales de los trabajadores de estrato marginal, vemos que se trata de carreras que se inician alrededor de los 14 años. En algunos casos están acompañados de la salida del hogar de origen, principalmente por problemas familiares. Se trata de inicios que, usualmente, están vinculados a la actividad desarrollada por los adultos de referencia en la adolescencia y juventud temprana, exceptuando algunos casos donde la entrada en la vida laboral coincide con la salida temprana del hogar de origen. En general se trata de recorridos que permanecen en un segmento secundario del mercado laboral. Sin embargo, entre estos trabajadores existen también trayectorias ascendentes, es decir que se trata de recorridos que, si 
De Prácticas y discursos/ Universidad Nacional del Nordeste/ Centro de Estudios Sociales

bien se iniciaron en la inestabilidad del segmento secundario, luego accedieron a un empleo bajo relaciones formales, aunque al momento de la entrevista, en un tercer tiempo de su trayectoria, se encontraban realizando nuevamente un empleo en el sector informal.

Por su parte, entre los trabajadores con origen medio/bajo integrado, el ingreso al mercado laboral se da en el marco de un trabajo asalariado de baja calificación en un establecimiento formal. Coincide con un ingreso más tardío que, sin embargo, con algunas excepciones, no implica la finalización del nivel secundario. En este sentido, los accesos al primer empleo estuvieron movilizados por el trabajo de los padres $u$ otros conocidos, marcando la prevalencia de los lazos sociales por sobre las credenciales educativas como recurso.

Si bien entre estos trabajadores las trayectorias tienden a ser de permanencia en el sector primario, existen casos con recorridos ascendentes -se trata de trabajadores que tuvieron sus primeros empleos en el marco de trabajo asalariado en establecimientos informales-. Por último, encontramos, aunque en una proporción minoritaria, trayectorias estabilizadas en el sector secundario o que descendieron hacia el mismo, aunque han experimentado alguna inserción -aunque breve- en un empleo formal.

Tabla 1: Contenidos de las trayectorias al año 2014 por origen social 


\begin{tabular}{|c|c|}
\hline \multirow[b]{2}{*}{$\begin{array}{c}\text { Origen } \\
\text { popular } \\
\text { vulnerable }\end{array}$} & \\
\hline & 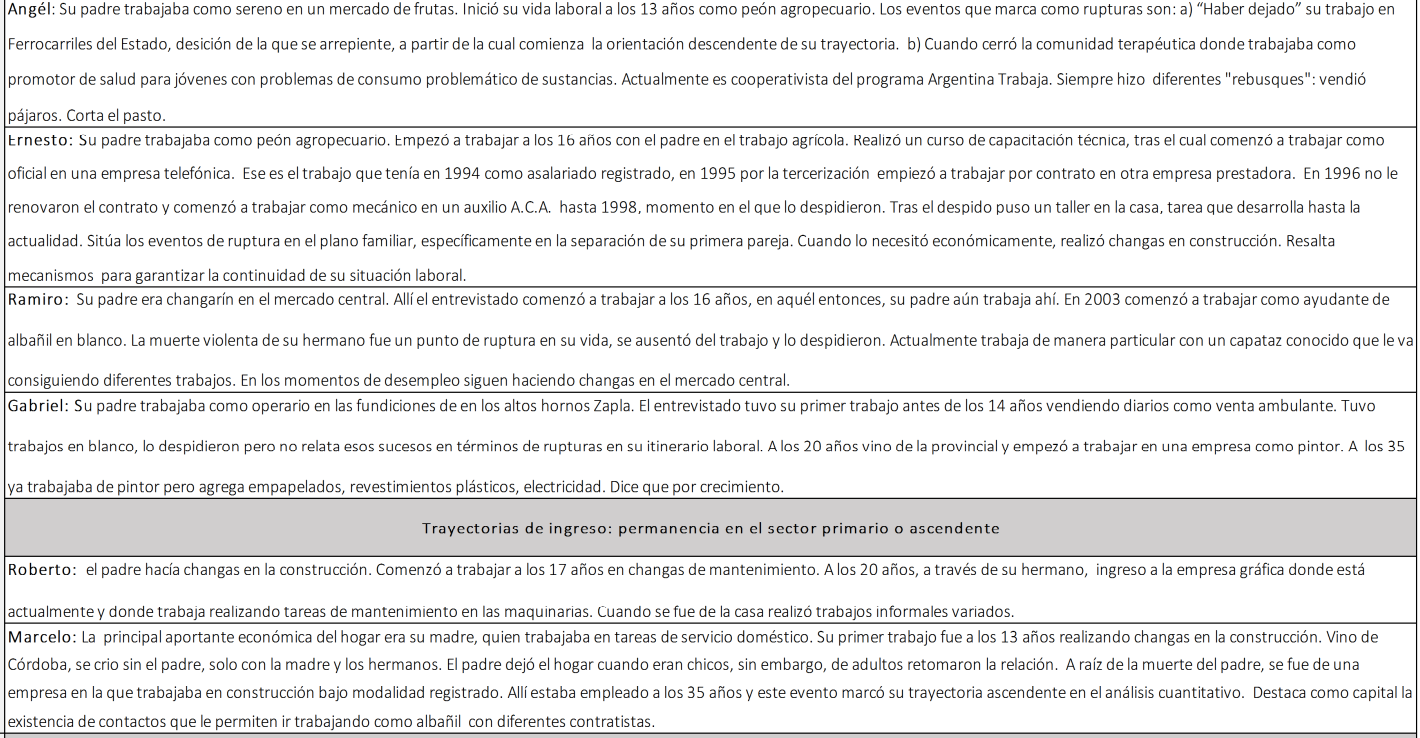 \\
\hline $\begin{array}{l}\text { Origen } \\
\text { popular } \\
\text { medio }\end{array}$ & $\begin{array}{l}\text { Actualmente es vigilador, sus ingresos son bajos, si bien tiene un empleo formal, se identifica con los trabajadores de bajos ingresos. } \\
\text { Martín: Su padre era asalariado formal en una fábrica de maquinarias. Comenzó a trabajar a los } 18 \text { años de edad, como operario en Danone/ La Serenisima, por recomendación. Dejo de vivir con sus } \\
\text { padres a los } 18 \text { años de edad, momento en que inició su vida conyugal y tuvo a su primer hijo. En } 2006 \text {, pasa de ser operario a maquinista, posición que conserva en la actualidad } \\
\begin{array}{l}\text { Manuel: Su padre tenía un quiosco de Diarios. Empezó trabajando antes de los } 14 \text { años como ayudante del padre,por medio de su hermano, consiguió trabajo como chofer de distribuidora láctea. Si bien } \\
\text { en un incio trabajó sin registro, luego pasó a ser asalariado formal. Continúa en ese trabajo hasta } 2014 \text {. }\end{array}\end{array}$ \\
\hline
\end{tabular}

\subsection{Las trayectorias desde una mirada interpretativa: valoraciones sobre la} situación laboral y sobre los mecanismos de movilidad

Abordamos la evaluación de los actores sobre su situación laboral individual al momento de la entrevista. En este sentido, cabría esperar que los trabajadores provenientes de origen medio/bajo integrado tengan valoraciones positivas sobre su vida laboral en tanto tienen mayores chances de tener carreras asociadas al segmento primario del mercado de trabajo. En segundo lugar, indagamos sobre la existencia de mecanismos de movilidad y/o reproducción socio-laboral, intentando relevar de manera comprensiva las modalidades y prácticas a partir de las cuales los entrevistados estructuran su vida laboral presente y proyectan su propio futuro laboral y el de sus hijos. En este punto sería esperable que las representaciones tiendan a reproducir las lógicas de segmentación laboral señaladas en el apartado anterior. Sin embargo, la 
De Prácticas y discursos/ Universidad Nacional del Nordeste/ Centro de Estudios Sociales

construcción de subjetividades no es únicamente racional, también es resultado contradictorio entre las condiciones materiales de los sujetos y la internalización que ellos hacen de las mismas, adjudicándoles sentido (Batittisni, 2009). En la misma línea, la construcción identitaria que tiene lugar a través de un relato de vida (Demaziere y Dubar, 1997) no responde a criterios de verdad o falsedad de las afirmaciones, sino a un análisis de los mecanismos de producción de sentido que se hacen presentes en el relato a través de un conjunto de articulaciones que los estructura (Kornblit, 2007).

La hipótesis de trabajo que orienta esta sección señala que: la cercanía entre la posición social de origen y las características que asume el proceso de inserción al mercado de trabajo serían relativizadas en el plano de los significados a partir de los cuales los actores leen sus elecciones y su situación laboral presente. Es decir que no necesariamente observaríamos en el plano subjetivo la existencia de chances de inserción más precarias y el endurecimiento del origen social sobre la calidad del

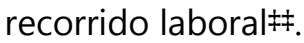

Con fines comparativos hemos fusionado el análisis de los relatos en tres categorías de recorridos, en las que se agrupan la mayoría de las entrevistas que hemos identificado en la base de datos cuantitativa: las trayectorias de origen marginal y las trayectorias de origen medio/bajo integrado. Entre las últimas, tomamos aquellas que experimentaron un ingreso al mercado laboral ascendente o estabilizado en el segmento primario, mientras que entre las primeras consideramos las del segmento secundario o descendente y aquellas que experimentaron movilidad hacia el sector primario.

Origen en el estrato marginal: Trayectorias de ingreso: permanencia en el sector secundario o descendente

Estos casos iniciaron su vida laboral en el trabajo de venta ambulante, realizando changas o siendo peones, luego se volcaron a la realización de un oficio que

\footnotetext{
\# Es importante señalar que esta hipótesis no desconoce que, aunque a nivel subjetivo se debilita su incidencia, el mundo de significaciones que los trabajadores constituyen también es producto de las condiciones materiales, que en nuestra propuesta de análisis están asociadas a las chances reales de movilidad entre el origen social y los recorridos de ingreso al mercado laboral. Pero se trata de percibir también las contradicciones presentes entre ambos planos.
} 
De Prácticas y discursos/ Universidad Nacional del Nordeste/ Centro de Estudios Sociales

desempeñan hasta la actualidad. Con la excepción de un entrevistado, que trabajaba en una cooperativa del programa Argentina Trabaja y que organizó su trayectoria laboral con changas en diferentes actividades, el resto se encontraba, al momento de la entrevista, desarrollando diferentes oficios: pintor, albañil y mecánico.

Los casos que han ocupado posiciones en el segmento primario plantean, a nivel subjetivo, dos formas de vinculación con el empleo registrado. Por una parte, los trabajadores que lo sitúan en un marco de mayores oportunidades y bienestar material, en estos casos la pérdida del empleo en el segmento primario del mercado de trabajo se interpreta como un viraje en sus trayectorias que implicó una pérdida, tanto en términos materiales como simbólicos. Otros casos en cambio accedieron al sector primario de manera ocasional y no perciben ese episodio como un momento de viraje, por el contrario, resaltan su continuidad en el trabajo de oficios -pintor y mecánicocomo una cualidad en su trayectoria. Aun tratándose de trayectorias que fueron definidas desde lo estructural como descendentes, las salidas del segmento primario no son relatadas en un sentido negativo, por el contrario, los inicios laborales suelen ser reivindicados como instancias de aprendizaje del oficio que inclusive se presentan opacadas por la necesidad de tener que trabajar bajo relación de dependencia debido a la falta de experiencia.

Gabriel: Sí, cuando empezaba sí, cuando empecé sí, con una empresa, Tremec se llamaba la empresa, cuando vine de la provincia. Yo vine de Jujuy a los 20 años acá, ahí sí empecé, trabajé un año, dos años, no me acuerdo bien cuánto trabajé.

Entrevistadora: En blanco.

Gabriel: En blanco, y de ahí me rajaron y arranqué solo, porque yo el oficio lo sabía bien, así que no...

Entrevistadora:¿Cómo aprendió usted el oficio?

Gabriel: Mi tío es pintor, o sea que yo mamé todo eso, yo tenía 15 años y era oficial. Yo cuando vine acá el oficio ya lo sabía, pero yo para arrancar acá tuve que trabajar para un patrón...

Entrevistadora:¿Y no quería eso?

Gabriel: No, porque yo el oficio lo sé, ¿por qué yo tengo que trabajar para que otros ganen plata? Porque yo trabajando por mi cuenta, como yo trabajo en mi trabajo, yo gano plata, yo, 
De Prácticas y discursos/ Universidad Nacional del Nordeste/ Centro de Estudios Sociales

pero porque yo trabajo, trabajo bien, me sacrifico y veo la ganancia. (Entrevista a Gabriel: origen en el estrato marginal, trayectoria precaria).

El saber del trabajo independiente, fuertemente asociado con las representaciones sobre la libertad en el empleo y con el manejo todos los ingresos, funciona como una diferenciación respecto a otros tipos de cuentapropismo. La construcción de la imagen que asocia el oficio con las estrategias reproductivas está enlazada con la actividad laboral como medio de vida que permite que ellos y sus familias puedan "vivir bien".

Entrevistadora: ... y usted, ¿cómo cree que está desde el 2008?

Gabriel: Crecí, mi familia creció, creció en el sentido de vivir bien y bueno, la familia también porque ahora tengo una nieta, pero sí creció en el sentido de que uno gana más, yo gano más, aunque las cosas están más caras viste, pero como te digo, yo vivo bien... Alcanza, alcanza, vivimos bien, vivimos bien. Yo no me quejo... siempre me quejo de la gente que dice que no tiene trabajo, no tiene trabajo porque no quiere trabajar.A mí la vida me enseñó a ganarme el pedazo de pan de la forma que sea, yo cortaba el pasto para ganarme un plato de comida, hoy cualquiera puede hacer lo mismo; si alguien me dice "mirá te corto el pasto, necesito para comer 20 pesos, córtame el pasto". Por eso te digo, yo estoy bien, yo no me quejo, cuando no tengo trabajo, llamo a mis amigos que tienen trabajo y les pido trabajo.

Entrevistadora: ¿Y qué hacen sus amigos?

Gabriel: Trabajan; o sea, yo tengo amigos que trabajan como yo, viene a ser como contratistas; cuando a mí el trabajo se me afloja, ya no voy a tener nada, los llamo y bueno, me dicen: vení que hay algo, voy, trabajo con ellos hasta que lo mío se normalice y sigo trabajando. (Gabriel: origen en estrato marginal, trayectoria precaria).

En otros casos, la valoración positiva sobre el oficio aparece debilitada cuando el entrevistado reconoce un punto de viraje en su trayectoria laboral, ese quiebre está vinculado al abandono o al despido de algún empleo -aunque de baja calificación al interior del segmento primario-.

Ramiro: [...] yo estoy, siempre igual, 11 años que estamos juntos nosotros (con la cónyuge) y bueno... y tuve, tuve mi trabajo bueno, pero no lo aproveché...pasa que tuve un problema, falleció mi hermano hace 2 años, me mataron a mi hermano y... me agarro mal, me cayó mal y perdí el trabajo por faltar, falté 15 días seguidos y bueno, me echaron... 
De Prácticas y discursos/ Universidad Nacional del Nordeste/ Centro de Estudios Sociales

Ramiro: Sí, ahí perdí el trabajo y no, desde ese momento se me hizo muy difícil conseguir de vuelta, y no puedo conseguir. (Ramiro, origen en estrato marginal, trayectoria precaria).

En estos relatos los sentidos atribuidos al trabajo actual están asociados a la salida de un empleo registrado como una ruptura en su trayectoria.

Ángel: Era poco, pero estaba en blanco, tenía aguinaldo, todo, no era mucho, pero había. En el ferrocarril sí, cometí el error de irme cuando cobrábamos dos mangos, me fui por un laburo mejor que duré 1 año en la fábrica de pastas. Viste, salís de acá por probar y pum, y perdés en otro lado. En el ferrocarril si estuviera ahora estaría ganando, no sé, no te digo 15 mil pesos, pero 12 mil por ahí, lo que está cobrando ahora un guardabarrera. Yo era guardabarrera, tenía prácticas de boletería, mi hermano laburaba en Constitución, el que vive acá al lado también era ferroviario y se fue con retiro voluntario con perdón de la palabra, ese error boludo, yo no, me fui, renuncié, por lo menos agarré unos mangos. (Ángel, origen en estrato marginal, trayectoria precaria)§§.

Estas rupturas están enlazadas con cargas de sentido positivas respecto al trabajo formal. Además, las condiciones de trabajo actual -principalmente las asociadas al ingreso- son consideradas, en estos casos, como limitaciones a sus estrategias de reproducción familiar.

Ángel: ...es que se me estancó la casa cuando trabajaba en la comunidad y me indemnizaron. Ella [la cónyuge] trabaja un poco mejor y me ayudaba un montón. Y ahora no hay entrada, no hay entrada, lo que está hecho, está hecho hace años, es una cosa que no podemos. La otra vez se nos rompió la heladera, juntando manguito sobre manguito compramos otra, la estamos pagando todavía, pero no podés progresar, no porque es una entrada, es una entrada, cómo subsistir con esa entradita, ¿viste? (Ángel, origen en estrato marginal, trayectoria precaria).

La idea de que ese tipo de inserción se perdió de manera definitiva en la trayectoria individual convive con expectativas activas con relación a la inserción laboral de los hijos. Asociado a esto, aparece la idea de la educación como canal de acceso al trabajo

\footnotetext{
${ }_{\S}$ Ángel menciona otra ruptura: el cierre de la comunidad terapéutica donde trabajaba. Actualmente es cooperativista del programa Argentina Trabaja y su recorrido laboral reciente está marcado por una trayectoria en programas de empleo y la realización de otras actividades transitorias, sobre todo desde la salida del segundo episodio de trabajo formal.
} 
De Prácticas y discursos/ Universidad Nacional del Nordeste/ Centro de Estudios Sociales

formal. Esta percepción suele estar, a su vez, relacionada con la imagen de que su propia trayectoria fue coartada por la interrupción de los estudios formales.

Ángel: No sé, yo lo que quiero es un futuro para ellos, que estudien, que trabajen, que tengan un trabajo, que no tengan que pasar lo que yo tengo que pasar, andar pichuleando, con un trabajito y con otro. Eso, un buen trabajo para ellos nada más, después Dios dirá lo que va a pasar conmigo más adelante, pero mientras ellos estén bien, voy a estar bien yo. (Ángel, origen estrato marginal, trayectoria precaria).

Ramiro: Yo se lo voy a exigir que me termine el secundario, ahora de ahí en adelante que él después se fija lo que quiere hacer, ya va a estar bastante grande. (Ramiro, origen estrato marginal, trayectoria precaria).

En el caso de las trayectorias de los trabajadores de oficios, el abandono educativo no es representado como descapitalización en la trayectoria individual, pero es considerado central para la movilidad de los hijos, para quienes en muchos casos se aspira al trabajo en blanco.

Entrevistadora: Y su hijo, Diego, ¿desde cuándo trabaja con usted?

Gabriel: Y desde los 16 años, abandonó el colegio y a veces trabaja conmigo, y a veces trabaja por ahí, pero... no, no quiere estudiar más, ¿viste? Y bueno, cada uno elige lo que quiere, ¿no?

Entrevistadora:¿No quiere volver a estudiar?

Gabriel: No, se lo planteé yo y me dijo: "No, no quiero".

Entrevistadora:¿Qué le dijo usted?

Gabriel: Que vuelva a estudiar, me dijo que no, que por ahora no tiene ganas, es grande... 21 años es grande, sabe lo que quiere, yo no lo voy a poner... no lo voy a crucificar para que se ponga a estudiar, si él no quiere, no quiere.

Entrevistadora:¿Pero usted preferiría que estudie?

Gabriel: Y sí, por el trabajo, hoy por hoy necesitás la secundaria completa para tener un buen trabajo. No un buen trabajo, un trabajo normal, en blanco, pero hoy por hoy los pibes son muy rebeldes, ¿viste? No lo entienden, lo va a entender cuando tenga 30 años, por ahí. Yo hay cosas que lo entendí cuando tenía 30 y lo entendí cuando tenía 40; o sea, son etapas, ¿viste? Uno va quemando etapas.

Entrevistadora: Es importante para usted que él pueda conseguir...

Gabriel: Un trabajo fijo.

Entrevistadora:En blanco. 
De Prácticas y discursos/ Universidad Nacional del Nordeste/ Centro de Estudios Sociales

Gabriel: A mí me encantaría, a mí sí, para él sí.

Entrevistadora:¿Porqué para él sí y para usted no?

Gabriel: Porque a mí me gusta el trabajo mío y a él no; si a él le gustaría el trabajo mío, estaría trabajando conmigo, entendés. Estaría buscando trabajar en otro lado. Por eso te digo, hay gente que le gusta y hay gente que trabaja por necesidad en la pintura, trabaja por necesidad en la albañilería, trabaja por necesidad en otra cosa, por necesidad, porque no saben el oficio, el que sabe el oficio, porque le gusta el oficio.

Entrevistadora: En cambio su hijo...

Gabriel: No, no es lo mismo. Por eso te digo, me gustaría que trabaje en un lugar fijo y tenga su sueldo, y sea responsable. Lo que pasa es que trabajar en otro lado, se manejan distinto a la forma que se trabaja conmigo, porque trabajar conmigo es una forma, es como que podés llegar tarde, como que yo lo llevo al trabajo, como que yo lo traigo, y en otro lado no y eso lo tiene que ver él, sino no lo va a ver nunca, entendés... y yo le dije "conseguite un laburo en otro lado, así vas a ver la diferencia que hay". (Gabriel: origen estrato marginal, trayectoria precaria).

Volviendo sobre las hipótesis señaladas más arriba, una mirada interpretativa nos mostró que: en el oficio funciona como un saber vinculado a la posibilidad de organizar el trabajo y trabajar de manera independiente a través del desempeño de estrategias para garantizar la continuidad laboral. Estas representaciones anulan las percepciones sobre las chances de inserción precaria en las que puede desarrollarse su actividad. Sin embargo, entre aquellos trabajadores que han experimentado las salidas de la formalidad como rupturas en sus carreras surgen las relaciones negativas entre el pasado y el presente laboral. En estos casos, la precariedad de la inserción actual está asociada a las elecciones pasadas, en términos de decisiones vinculadas a la pérdida de oportunidades.

Como puede observarse, estos dos tipos de trayectorias tienen en común el origen social y el origen laboral; sin embargo, se trata por un lado, de trayectorias ligadas a carreras de oficio y por otro de trayectorias que, ya sea desarrollando o no un oficio en la actualidad, son percibidas como truncas. Ambas resultan divergentes con relación a los sentidos que las personas adjudican a su situación actual y a las estrategias de movilidad laboral: en un caso están asociadas con la puesta en juego de diferentes destrezas para mantenerse de manera activa en el oficio y en otro aparecen, por fuera 
De Prácticas y discursos/ Universidad Nacional del Nordeste/ Centro de Estudios Sociales

de la propia trayectoria, únicamente asociadas a las expectativas de movilidad laboral para los hijos.

\section{Origen en el estrato marginal: Trayectorias de ingreso: ascendentes o permanencia en el segmento primario}

Se trata de casos que, desde la tipología cuantitativa, suponen un recorrido laboral ascendente, ya que se iniciaron en una actividad de baja productividad en el sector secundario y en el promedio de su vida activa alcanzaron un empleo en el segmento primario. Sin embargo, las valoraciones sobre el trabajo no siguen necesariamente esta dirección.

El presente laboral, anclado en el sector primario a través de la realización de un trabajo manual asalariado, se vincula con los eventos laborales previos informales desde la salida del hogar de los padres. En estas trayectorias más verticales, el ingreso al establecimiento formal no necesariamente aparece como un punto de viraje. Roberto, operario que trabaja manejando una máquina en una papelera, no está conforme con su trabajo, al que define como rutinario y aburrido. Por otra parte, su relato marca su salida del sistema educativo y su ingreso temprano al mercado laboral como un evento que influenció el rumbo de su carrera laboral, donde las condiciones de vida de su familia aparecen como centrales. Cabe destacar que, aunque desde hace varios años tiene ese trabajo, no muestra identificación con ese contexto laboral, buscando estrategias que le permitan revertirlo por fuera del mismo.

Roberto: Yo empecé a laburar de muy chico, 13 años, ya estaba laburando. Entonces se complicaba, en mi caso. Mi viejo era alcohólico, mi vieja trabajaba sola, y se complicaba un poco el tema, ¿viste? Entonces tomé la decisión y dije "me voy", y así salió un trabajo. Y así cambiando de trabajo en trabajo.

Entrevistadora: ¿Y desde cuándo estás en este trabajo?

Roberto: Sí, desde el 2001. Y bueno, mucho antes trabajé en curtiembres, construcción, fábrica de copas...y bueno, pasó el tiempo y no pude retomar más [los estudios] y bueno, ahora se hace todo más difícil... pero pienso "es un año solo". Ya pasó medio año, me queda medio año más. Si hice otros cursos que fueron más complicados, cómo no voy a hacer este. 
De Prácticas y discursos/ Universidad Nacional del Nordeste/ Centro de Estudios Sociales

Entrevistadora: Claro, estás terminando el secundario.

Roberto: Claro.

Entrevistadora: Y eso, ¿cuándo lo decidiste?

Roberto: A principio de este año. Me faltaba $5^{\circ}$ año y decidí terminar. (Roberto, origen estrato marginal, trayectoria no precaria).

Sus estrategias de cambio están movilizadas por la educación que aparece como la principal referencia de su recorrido biográfico en términos de limitación. Valora la educación como recurso para modificar su carrera laboral.

Entrevistadora: Y eso, vos con el título, te facilitaría entrar a trabajar en...

Roberto: Sí, sí. Yo, por ejemplo, estoy haciendo esto porque yo quiero ser chofer de colectivos. $Y$ entonces al no tenerlo, bueno, busqué los medios rápidos para entrar. Entonces mientras pasa este año voy haciendo los requisitos que me piden. Pero es una posibilidad, después veremos qué es lo que sale. También el trabajo, me gusta el trabajo, pero es algo que me lleva mucho tiempo. Es como que me aburre, ya está, hace 12 años que estoy ahí. Me lo sé todo de memoria. Entonces quiero salir de ahí, me aburre. Me gusta manejar, entonces... ver de salir de ahí. Ahora está semanita estoy de vacaciones, voy a ver si hago estudios, registros, psicólogo, la Policía de Capital, bueno, juntando cosas. (Roberto, origen estrato marginal, trayectoria no precaria).

Roberto valora su trabajo actual en tanto medio para avanzar hacia otro tipo de empleo, pero lo reconoce como un recurso que le permite avanzar en ese sentido. Marcelo, trabajador de la construcción, también tuvo una trayectoria de ingreso ascendente, pero no pudo mantenerse en la misma y al momento de la entrevista se encontraba realizando tareas de albañil para diferentes contratistas. En su caso, la salida del empleo registrado es percibida como una ruptura en su trayectoria laboral porque estuvo vinculada con la muerte de su padre.

Entrevistadora: ¿Cambió algo desde aquel momento [2008, año del relevamiento de la encuesta] hasta acá en relación a tu familia, vos seguís en el mismo trabajo?

Marcelo: No, yo el trabajo lo dejé por un tema mío, personal. Porque mi papá laburó mucho tiempo ahí en la empresa y falleció, y como me dieron un poquito la espalda yo renuncié. No 
De Prácticas y discursos/ Universidad Nacional del Nordeste/ Centro de Estudios Sociales

me gustó el trato que le dieron, tantos años que estuvo de capataz, qué se yo. Cuando yo fui a solicitar una mano cuando más lo necesité, nunca pedí nada en la empresa, siempre obré por la mía, yo no quería que me regalen nada, yo quería que me ayuden en ese momento nada más, te hacen unos descuentos, yo por eso me fui, el trabajo sigue, pero ellos... Yo solamente falté y falté para que me den la cosa, mandé la carta, ellos me rechazaron la carta, pero me dijeron: para que vos cobres el fondo de desempleo, qué se yo, te mandamos que te echamos. Es más, ni eso hice, no quiero nada, no quiero nada. Agarré, me fui, conseguí un trabajo con un muchacho porque yo toda la vida laburé de construcción, yo tengo conocidos, tengo contactos con mucha gente, aparte sé el oficio, tengo posibilidades de salir y entrar en otro lado, entonces no me quedé, por eso me fui, no es porque me echaron; es más, mi hermano está ahí y yo... (Marcelo, origen estrato marginal, trayectoria no precaria).

La valoración de su trabajo actual está por sobre su experiencia formal. Si bien reconoce algunos beneficios, señala que los descuentos al salario eran altos y que durante su trabajo informal pudo avanzar en la construcción de su casa.

Marcelo: Estoy igual, estoy igual en el sentido plata, pero obvio cobro un poquito más, porque en la empresa me descontaban, qué se yo, y a acá no me pagan todo.

Entrevistadora: ¿Y te conviene eso?

Marcelo: Sí, por ahora me conviene, porque en 1 año, en ese año que estoy acá, levanté todo esto y yo cuando estuve allá, 7 años en la empresa, no podía hacer nada porque siempre tenía que pagar, pagar, pagar, me descontaban, no me quejo, porque cuando yo vine a vivir acá, no tenía nada, ni una silla tenía. Yo compré el terreno, pero sin nada, tenía esta casita sola. Y yo agarré con mi señora, con lo del día, con dos pilchas, una cama y vamos, vamos y así tenía mi casa.(Marcelo, origen estrato marginal, trayectoria no precaria).

Mientras el trabajo en una empresa con registro no aparece como referencia identitaria, los saberes que posee sobre su actividad de albañil y las relaciones sociales que ha podido movilizar en ese ámbito son fuertemente valorados. Las características de su actividad no son representadas como una limitación para el desarrollo de sus estrategias de vida. Pero esta idea surge, en cambio en relación al barrio de residencia y las chances de vida que ese entorno territorial brinda para el desarrollo de sus hijos. 
De Prácticas y discursos/ Universidad Nacional del Nordeste/ Centro de Estudios Sociales

Marcelo: La mejor herencia es el estudio, que tengan un buen estudio, por el momento se puede pagarlo y después se verá. Uno en su momento no pudo estudiar, por lo menos que ellos tengan la oportunidad.

Entrevistadora: ¿Y por qué se quiso cambiar?

Marcelo: Por el secundario, y aparte es como que en el barrio...todo se escucha lo que pasa en un barrio, ¿no? Y acá en el colegio este, hay cosas que no me gustan, yo quería que cambie de ambiente porque acá hay muchos chicos en la calle, mucha esquina hacen, mucha esquina hacen, y a mi hijo no quiero que haga eso, quiero que sea otra cosa y allá en Longchamps... ¿Cómo te puedo decir? Son más chetos, son más... (Marcelo, origen estrato marginal, trayectoria no precaria).

La valoración del trabajo registrado en estos relatos no surge como aspecto central de las condiciones de vida de estos trabajadores. La educación representa un sinónimo de movilidad, como un recurso para el cambio en la propia trayectoria o valorada, aunque de manera muy general como recurso para los hijos.

\section{Origen en el estrato medio/bajo integrado: Trayectorias de ingreso: permanencia en el sector primario a ascendentes}

Estos recorridos comparten con las trayectorias antes analizadas las características respecto a la permanencia en el sector formal, aunque las percepciones de los trabajadores sobre su situación laboral resultan más heterogéneas.

El primer caso se desarrolla bajo el paradigma tradicional asociado a las cadenas de movilidad del segmento primario. Martín empezó en la fábrica en la que actualmente trabaja a través de un conocido, fue ascendiendo posiciones, accede a capacitaciones, aunque también manifiesta realizar tareas rutinarias, con muchas horas de trabajo y con horarios rotativos. Actualmente se desempeña como maquinista. Valora positivamente la estabilidad, la obra social, la afiliación sindical y las posibilidades de acceder al crédito formal a través de la financiación que otorgan las tarjetas de crédito. Señala que un límite en su trayectoria es no haber finalizado la escuela técnica, la cual está terminando actualmente.

Entrevistadora: En algún momento, ¿pensó en cambiar de trabajo? 
De Prácticas y discursos/ Universidad Nacional del Nordeste/ Centro de Estudios Sociales

Martín: Bueno, vamos a decirlo de una manera, lo que pasa que uno trabajando en relación de dependencia, en horarios rotativos es como que uno no vive siempre a un ritmo del que quisiera, porque, por ejemplo, yo esta semana estuve de noche, eso ya te cambia un montón de cosas, qué se yo. Por decírtelo así, por hacer un análisis, desde el lunes que lo venía pensando mirá, lo hice recién ayer por un tema, es que yo llego, trabajo de 9 a 5.(Martín, medio/bajo integrado, trayectoria no precaria).

Entrevistadora ¿Cómo está usted con su trabajo, lo que piensa de su trabajo?

Martín: Eh... antes para mí era buena, hoy en día es buena, por ahí es algo que medianamente me gusta, la de antes por ahí era, si bien cambió la estructura, esa línea de producción cambió, como que ahora se hicieron inversiones dentro de esa estructura, cambió un poco de lo que era antes, era más manual, más artesanal, y hoy en día es un poquito, sigue siendo artesanal, pero ahora se maneja con una máquina, ahora es otra cosa.(Martín, origen medio/ bajo integrado, trayectoria no precaria).

El segundo caso inició su carrera laboral en una fábrica, años después perdió el trabajo e ingresó en la empresa de seguridad por un conocido de su padre, quien era policía. En su trayectoria parece regir una relación instrumental con los trabajos, siempre tuvo empleos formales y reconoce que a lo largo de su carrera ha tenido "pocos cambios", al mismo tiempo si bien no define su situación como mala, resalta el poco margen económico que tiene el hogar. Las valoraciones negativas sobre su trabajo están asociadas a la realización de tareas rutinarias. Los ingresos le permiten llegar a fin de mes, pero habilitan poco margen de acción en el hogar. Parte de las representaciones negativas sobre su trabajo están asociadas con la salud: el empleo es estable, pero requiere buenas condiciones físicas, ya que tiene exigencia, rotación de turnos, etc. Sin embargo, no rastreamos en su relato la idea del empleo registrado como ideal, es más: manifiesta conocer gente que trabaja sin registro por su cuenta y accede a mejores ingresos.

En su trayectoria aparece la educación como aquello que sus padres no pudieron brindarle y que valora para sus hijos. Cabe destacar que no es concebida en términos instrumentales, sino que el entrevistado el asigna un valor moral ligado a lo vocacional, 
De Prácticas y discursos/ Universidad Nacional del Nordeste/ Centro de Estudios Sociales

vinculándola a un aspecto moral de superación y no como un recurso laboral. No aparece de manera en su discurso la vinculación entre educación y trabajo.

Entrevistadora: Antonio, yo me quedé pensando en algo que usted dijo, ¿qué le gustaría ser? "Yo soy vigilador porque es lo que hay...".

Antonio: Eh, bueno... lo que a mí me hubiera gustado ser es lo que le estoy inculcando a mi hijo; o sea, él me dice... "Vos lo que no pudiste hacer querés que lo haga yo", siempre dicen eso, es típico cuando se ponen rebeldes. Yo digo es para tu futuro, yo sigo; o sea...lo que a mí me hubiera gustado estudiar es música, por eso yo le compré una guitarra eléctrica a él, le gusta. Por lo menos lo sacó de la calle y va a tomar clases; o sea, es un pibe muy introvertido, no es de andar en la calle, siempre está ahí o con la computadora, ¿viste?Lo que más me gusta hacer es ser músico; o sea... Pero nunca mis padres tuvieron... este... dinero como para mandarme a estudiar o comprarme. (Antonio, origen estrato medio/bajo integrado, trayectoria no precaria).

Martín: Yo pienso que tienen que estudiar porque yo no es que uno que la grabadora de mi mamá, de mi papá, pero "vos tenés que estudiar no porque no seas alguien, sino para que tengas un mejor pasar".

Entrevistadora: $Y$ estudiar significa ¿terminar el secundario o seguir estudiando?

Martín: Seguir estudiando, yo no lo terminé al secundario, pero fui a un industrial. No lo terminé, pero estoy arrepentido y no...pero bueno, pero para ellos que sea distinto, digamos, qué se yo, que pasen esa etapa que yo no pasé, que la superen, que sean, qué se yo. No te pido que sean abogados, ingenieros, que tengan algo que digan: bueno, puedo trabajar de tal cosa que no, porque ellos me dicen vos trabajás por cantidad de días, no estás con nosotros, ¿entendés? (Martín, origen estrato medio/bajo integrado, trayectoria no precaria).

El otro entrevistado comenzó su trayectoria en la empresa donde se desempeña actualmente como camionero. Su recorrido en el segmento primario parece influir en que no tenga una perspectiva aspiracional del trabajo formal. Considera las credenciales educativas como medio que permitiría a sus hijos una mejor situación laboral respecto a la que el propio entrevistado experimenta. Es decir, la valora como 
De Prácticas y discursos/ Universidad Nacional del Nordeste/ Centro de Estudios Sociales

un canal para que puedan acceder, aún en el sector primario, a mejores condiciones de trabajo.

No tiene un reconocimiento particular sobre los saberes que implica su trabajo, aunque posee una alta valoración respecto a la independencia en la actividad laboral. En este caso, surge la idea de profesión ligada a una independencia que el entrevistado no posee en su relación laboral. De esta manera, su propia trayectoria se vincula con las representaciones sobre los mecanismos de movilidad laboral para sus hijos.

Manuel: Voy a tratar por todos los medios que estudie, pero no por mí, a mí no me interesa, yo ya estoy; o sea, yo siempre les digo a ellos, yo soy un mulo, sos una mula que trabajás para un tipo, que tenés que hacer lo que te dice el tipo, si no hacés eso, te tenés que ir, es así, funciona así. Entonces porque vos vas a ir por el mismo camino, si vos tenés la opción de estudiar, estudiá, sé algo y trabajás para vos, que vos puedas decidir lo que vas a hacer.(Manuel, origen medio/bajo integrado, trayectoria no precaria).

El análisis interpretativo de los casos nos muestra que los logros educativos aparecen como un elemento con incidencia en la construcción de las expectativas. Surge en este punto una diferenciación, ya no vinculada al tipo de ingreso que los entrevistados tuvieron en el mercado de trabajo, sino en relación a cómo valoran sus recorridos. Entre los que desarrollaron una trayectoria de oficio, las potencialidades de la formación no están ligadas a la escolaridad, pero entre quienes añoran la inserción formal perdida o están actualmente como empleados en una empresa formal, aparece como un elemento asociado a la puesta en marcha de mecanismos de movilidad. Si bien la referencia a la educación como medio de movilidad es transversal a los tres tipos de recorridos analizados, podemos señalar que, a medida que las trayectorias resultan más integradas surge el reconocimiento de la educación como factor de movilidad y, por lo tanto, como elemento aspiracional en torno a las expectativas de movilidad para los hijos.

El análisis de los tres tipos de recorridos nos lleva a relativizar el reflejo entre estructura y subjetividad. Creemos que esto constituye la fortaleza que presenta la posibilidad relacionar la perspectiva longitudinal bajo dos registros. Por una parte, uno material, vinculado a la reproducción de posiciones laborales al interior de un 
De Prácticas y discursos/ Universidad Nacional del Nordeste/ Centro de Estudios Sociales

segmento con un nivel de institucionalización determinado y, por otra parte, en un nivel simbólico que permite seguir las reconstrucciones subjetivas que dan un significado global a la trayectoria experimentada por cada individuo.

En este sentido, hemos notado que el origen social parece no tener peso en el marco de las representaciones sobre el trabajo, al menos cuando hablamos de orígenes que, aunque diversos, son jerárquicamente cercanos, como es el caso de la selección de trabajadores aquí realizada. En segundo lugar, podemos decir que la segmentación no es reflejada de manera lineal en el plano subjetivo. Es decir, no hay correspondencia entre una inserción no precaria o precaria y las representaciones y expectativas positivas en el plano laboral. Más bien lo contrario, las valoraciones más positivas sobre el pasado y el presente laboral aparecen en aquellas inserciones que son precarias en términos de segmentación, independiente del tipo de ingreso y del origen social de los trabajadores. De manera opuesta, quienes experimentan empleos en el sector primario suelen a la vez que reconocer los beneficios de su situación, expresar su disconformidad con algún aspecto de las condiciones laborales. Sin embargo, el factor educativo, que sin dudas constituye un aspecto relevante en los procesos segmentación del mercado laboral ${ }^{\star \star *}$ parece ser el único que mantiene su peso.

\section{Conclusiones}

En este trabajo nos propusimos observar los cambios en los procesos de reproducción social del trabajo. Los datos mostraron, por una parte, la existencia de un proceso de polarización dentro del cual las generaciones más jóvenes, que atravesaron su entrada al mercado de trabajo durante el proceso de apertura y ajuste económico,

\footnotetext{
*** Beccaria y Grosiman (2008) han señalado que la expansión del sector informal expresa, en parte, la baja capacidad de la economía formal de generar puestos de trabajos. Por eso, este tipo de inserciones tiende a concentrarse entre los trabajadores de baja calificación, ya que el sector formal asigna prioridad al nivel educativo como criterio de selección. Sin embargo, esto convive con otra dinámica reconocida: no obstante, los jóvenes acceden a niveles educativos más altos que los de sus predecesores, ello no repercute sobre la posición socio-ocupacional que los mismos ocupan (Jorrat, 2011).
} 
De Prácticas y discursos/ Universidad Nacional del Nordeste/ Centro de Estudios Sociales

tendieron a desarrollar en mayor medida trayectorias asociadas al segmento secundario del mercado de trabajo. Por otra parte, se manifiesta un mayor nivel de reproducción de las condiciones de inicio de la vida laboral y de la posición social de origen. De esta manera, la incidencia de los factores de origen sobre la incorporación en diferentes tipos de recorridos laborales se intensifica, aumentando su relevancia la cercanía con la posición social de origen.

Cuando rastreamos la existencia de los ejes de segmentación que agrupan estos procesos desde la perspectiva de los trabajadores, vemos que, en el plano subjetivo, asumen importancia las diferentes modalidades y prácticas a partir de las cuales los entrevistados estructuraron su vida laboral, y que la trayectoria de entrada al mercado de trabajo y las condiciones de origen asumen una menor centralidad.

Al analizar las significaciones que tienen respecto al trabajo quienes han experimentado una relación laboral en el segmento primario durante todo su itinerario, y recurriendo a aquellas significaciones que es de esperar sigan motorizando este tipo de empleos más típicos, vemos que este tipo de representaciones asociadas a las condiciones de la relación laboral exceden su lugar como recurso de sobrevivencia, pero no habilitan referencias que vinculen a la actividad laboral con la satisfacción personal. En otras palabras, si bien existió una cadena de movilidad por la cual transcurren las carreras, este aspecto no parece ser dador de sentido a nivel subjetivo. Estos mecanismos no niegan la incidencia del origen social sobre los tipos de trayectorias, pero permitirían relativizar su causalidad con relación a la imagen que los trabajadores tienen sobre su trayectoria global y a los mecanismos de identidad que ellos construyen en torno a las mismas.

Las trayectorias de descenso solo tienen lugar a nivel subjetivo cuando se trata de caídas formuladas como rupturas en el relato. Estas trayectorias son las que conservan, a nivel discursivo, la carga de la dualidad entre uno y otro segmento, ya que las rotaciones que las constituyen y las significaciones que la fuerza de trabajo otorga al empleo en el sector primario y su distancia con el secundario se vuelven centrales en la construcción de las expectativas sobre su propio futuro laboral y, principalmente, sobre las de los hijos. 
De Prácticas y discursos/ Universidad Nacional del Nordeste/ Centro de Estudios Sociales

Finalmente, también en las trayectorias de descenso están los recorridos desarrollados por quienes definen su actividad en términos de oficios, donde los enunciados sobre el manejo de la actividad y saberes del trabajo independiente disuelven las vinculaciones con las condiciones sociales de origen,quedando las referencias al origen y al tipo de inicio en el mercado laboral dentro de las trayectorias que hemos denominado de ruptura.

Sin embargo, los sentidos construidos en torno al trabajo, como actividad productiva de las condiciones de vida, parecen mostrar con mayor firmeza la existencia de procesos de precarización entre aquellos trabajadores que, jóvenes durante las décadas de las reformas estructurales, experimentaron sus trayectorias de ingreso al mercado laboral. Transversales a las segmentaciones que hemos planteado en estas páginas, la falta de satisfacción con el trabajo que aparece fuertemente entre quienes se desempeñan en relación de dependencia, junto con las estrategias de quienes desarrollan un oficio, muestran que las dualidades en las relaciones productivas no se traducen en la producción de subjetividades, como sí lo hacen los procesos de desintegración socio-laboral a nivel coyuntural.

Por último, no debe perderse de vista que hemos realizado este estudio en un barrio popular situado en la periferia del Gran Buenos Aires donde, si bien las inserciones laborales son heterogéneas, existen patrones ligados a las condiciones de vida y de trabajo que asumen una fuerte impronta territorial y que deberían ser objeto de nuevas investigaciones. Pero bajo las características-socio territoriales de este espacio vimos que las condiciones de empeoramiento relativo, que el artículo abordó desde una doble perspectiva, parecen evidenciar con mayor intensidad los efectos del contexto de las políticas económicas de ajuste y de flexibilización laboral sobre los procesos de reproducción social del trabajo, poniendo en evidencia el rigor con el que estos influyeron sobre los itinerarios de los trabajadores que desarrollaron sus procesos de ingreso laboral durante esta etapa.

\section{Bibliografía}


De Prácticas y discursos/ Universidad Nacional del Nordeste/ Centro de Estudios Sociales

Altimir, O.;Beccaria, L. y González Rozada, M. (2002). La distribución del ingreso en Argentina, 1974-2000. Revista de La CEPAL, 78, pp 56-85. Santiago de Chile

Battistini, O. R. (2004). El trabajo frente al espejo: continuidades y rupturas en los procesos de construcción identitaria de los trabajadores. Editorial Prometeo

Beccaria, L. y Groisman, F. (2008). Argentina desigual. Universidad Nacional de General Sarmiento. Buenos Aires

BERTAUX, D. (1980) "El enfoque biográfico: su validez metodológica, sus potencialidades", Cahiers Internationaux de Sociologie, Vol. LXIX. Paris. Pp. 197-225.

Bourdieu, P. (1988). La distinción. Bases y criterios sociales del gusto Madrid. Editorial Taurus.

Busso, M.; Longo, M. E. y Pérez, P. (2014). La estabilidad-inestabilidad laboral de jóvenes argentinos desde una perspectiva interdisciplinaria y longitudinal. Cuadernos de Economía, 33, 399-420.

Dubar, C. (2000). El trabajo y las identidades profesionales y personales. Revista Latinoamericana de estudios del Trabajo, 7(13), 5-16.

Elder, G. H. (1998). The life course as developmental theory. Child development, 69(1), $1-12$.

Erikson, R.y Goldthorpe, J. H. (1992). The constant flux: A study of class mobility in industrial societies. USA: Oxford UniversityPress.

Gaete, T. y Soto, Á. (2012). Esta es mi trayectoria, este es mi trabajo: narrativas e identidad en el trabajo en Chile. Psykhe, 21(2), 47-59.

García Blanco, J. M. y Gutiérrez, R. (1996). Inserción laboral y desigualdad en el mercado de trabajo: cuestiones teóricas. Reis, 269-293.

González de la Rocha, M. (2004). De los"Recursos de la Pobreza" a la" Pobreza de Recursos" ya las" Desventajas Acumuladas". Latin American ResearchReview, 39 (1), 192-195.

Jacinto, C. (2010). La construcción social de las trayectorias laborales de jóvenes: políticas, instituciones, dispositivos y subjetividades. Teseo Editorial. Buenos Aires.

Jacinto, C. y H. Chitarroni (2010).Precariedades, rotación y movilidades en las trayectorias laborales juveniles. Revista Estudios del Trabajo, 39/40. Buenos Aires. 
De Prácticas y discursos/ Universidad Nacional del Nordeste/ Centro de Estudios Sociales

Jorrat, R. (2011).Diferencias de acceso a la educación en Argentina: 20032007.Lavboratorio Revista de Estudios sobre Cambio Estructural y Desigualdad Social, 24. Reactualizando los debates acerca de la estructura y la movilidad social. Verano 2011.

Kornblit, A. (2007). "Historias y relatos de vida". Metodologías cualitativas en ciencias sociales: modelos y procedimientos de análisis. Buenos Aires: Biblos.

Longo, M. E. (2004). Los confines de la integración social. Trabajo e identidad en jóvenes pobres.En Battistini, O. (coord.) El trabajo frente al espejo. Continuidades y rupturas en los procesos de construcción identitaria de los trabajadores. Buenos Aires:Editorial Prometeo.

Mallimaci, F. y Salvia, A. (2005). Los nuevos rostros de la marginalidad: la supervivencia de los desplazados. Editorial Biblos. Buenos Aires.

Molina Derteano, P. (2007). Juventud ¿Divino Tesoro? Trayectorias socio-laborales de jóvenes trabajadores de delivery. En Sombras de una marginalidad fragmentada. Aproximaciones a la metamorfosis de los sectores populares de la Argentina. Miño y Dávila. Buenos Aires.

Montero Casassus, C. M. (1998). El uso del método biográfico en el estudio de trayectorias sociales precarias.En Vargas, P. y Zamudio, L. (eds.) Los usos de la historia de vida en las ciencias sociales, Vol. 17. Anthropos Editorial. Barcelona.

Mora Salas, M. y De Oliveira, O. (2014) "Los caminos de la vida: acumulación, reproducción o superación de las desventajas sociales en México" en Revista Mexicana de Ciencias Políticas y Sociales. Universidad Nacional Autónoma de México. Nueva Época, año LIX, enero-abril 2014, pp 81-116.

Muñiz Terra, Leticia. (2008). La pérdida del trabajo petrolero: transformaciones laborales, materiales e identitarias. Avá, (12), 95-116.

Muñiz Terra, L.(2011). Carreras y trayectorias laborales: una revisión crítica de las principales aproximaciones teórico-metodológicas para su abordaje. Revista Latinoamericana de Metodología de las Ciencias Sociales, vol. 2, 1, pp. 36-65. 
De Prácticas y discursos/ Universidad Nacional del Nordeste/ Centro de Estudios Sociales

Neffa, J. y Panigo, D. (2009).El mercado de trabajo argentino en el nuevo modelo de desarrollo. Dirección Nacional de Programación Macroeconómica. Ministerio de Economía y Finanzas Públicas. Argentina del Bicentenario. Buenos Aires:Editorial Biblos.

Nun, J. (1969).Superpoblación relativa, ejército industrial de reserva y masa marginal. Revista Mexicana de Sociología, vol. 5, 2, México.

Otero, A. (2009). Procesos de transición a la vida adulta: un estudio cualitativo con jóvenes argentinos. (Tesis doctoral) Buenos Aires, Argentina. FLACSO-Argentina.

Panaia, M. (2006). Trayectorias de ingenieros tecnológicos. Graduados y alumnos en el mercado de trabajo. Buenos Aires: Miño y Dávila Editores.

Parrado, E. A. (2005). Economicrestructuring and intra-generationalclassmobility in Mexico. Social Forces, 84(2), pp. 733-757.

Perez, P. y Busso, M. (2015). Los jóvenes argentinos y sus trayectorias laborales inestables. Mitos y realidades.Revista Trabajo y Sociedad, 24, Verano 2015.

Pérez, P.; Deleo C. y Fernández Massi, M. (2014). Desigualdades sociales en trayectorias laborales de jóvenes en la Argentina.Revista Latinoamericana de Población, Vol. 7,13, 2013, pp. 61-89.

Pérez Sáinz, J. P. (2000). Globalización, informalidad y pobreza en América Central. En Carpio, Klein y Novacovsky (comps.) Informalidad y exclusión social. Buenos Aires:FCE/SIEMPRO/OIT.

Pérez Islas, J.A. y Urteaga, M.(2001). Los nuevos guerreros del mercado. Trayectorias laborales de jóvenes buscadores de empleo.En Pieck, E.Los jóvenes y el trabajo. La educación frente a la exclusión social. México: Coedición UIA, IMJ, UNICEF, CinterforOIT, RET y CONALEP, pp 333-354.

Piore, M. (1975).Notas para una teoría de la estratificación del mercado de trabajo.En Toharia, L. (1983) El mercado de trabajo: teorías y aplicaciones. Lecturas seleccionadas. Madrid: Alianza editorial.

Pries, L. (1993). Biografía y sociedad: Un enfoque integrativo e interdisciplinario. Anuario de El Colegio de Puebla. 
De Prácticas y discursos/ Universidad Nacional del Nordeste/ Centro de Estudios Sociales

Pries, L. (1996). ¿Institucionalización o desinstitucionalización del curso de vida? Biografía y sociedad como un enfoque integrativo e interdisciplinario.Estudios demográficos y urbanos, pp. 395-417.

Przeworski, A. (1982). La teoría sociológica y el estudio de la población: reflexiones sobre los trabajos de la Comisión de Población y Desarrollo de CLACSO. Reflexiones teórico-metodológicas sobre investigaciones en población, pp. 58-99.

Pinto, A. (1970). Heterogeneidad estructural y modelo de desarrollo reciente de la América Latina. Inflación: raíces estructurales. México: FCE.

Quijano, A. (1988). La nueva heterogeneidad estructural de América Latina. Nuevos temas, nuevos contenidos. Caracas: UNESCO/Nueva Sociedad.

Roberti, E. (2014). La nueva condición juvenil: reflexiones sobre los sentidos y prácticas que configuran las trayectorias laborales de jóvenes pobres.Cuestiones de sociología, (11), pp. 1-20.

Ryder, N. B. (1965). The cohort as a concept in the study of social change. American sociologicalreview, pp. 843-861.

Salvia, A. y Chávez Molina, E. (2002). Estudios diacrónicos de varones beneficiarios del seguro de desempleo y pago único. Instituto de Investigaciones Gino Germani, Facultad de Ciencias Sociales, Universidad de Buenos Aires.

Salvia A.; Persia J.; De Grande P. (2000).Los Senderos del Desempleo: Una nueva Institucionalidad Social. Estudios sobre Trayectorias Socio-laborales de Desocupados en el Gran Buenos Aires. IV Simposio Internacional: el Cono Sur: su inserción en el tercer milenio, Facultad de Ciencias Económicas.

Salvia, A.; Vera, J. y Poy, S. (2015). Cambios y continuidades en la estructura ocupacional urbana argentina. EnLindenboim, J. y Salvia, A.(coords.) Hora de balance: proceso de acumulación, mercado de trabajo y bienestar en Argentina. Buenos Aires: EUDEBA.

Sautu, R. (2011).Acerca de lo que es y no es investigación en ciencias sociales.En Wainerman, C. y Sautu, R. (comps.)La trastienda de la investigación. Buenos Aires: Editorial Manantial. 
De Prácticas y discursos/ Universidad Nacional del Nordeste/ Centro de Estudios Sociales

Año 4, Número 5, 2015

ISSN 2250-6942

Anexo metodológico

\begin{tabular}{|l|l|l|}
\hline \multicolumn{3}{|c|}{ Trayectorias de inserción al mercado de trabajo } \\
\hline \multicolumn{2}{|c|}{ Concepto } & \multicolumn{1}{|c|}{ Indicadores } \\
\hline Se considera al proceso de inserción en el & Segmento de inserción de la ocupación del primer \\
mercado de trabajo a la etapa que transcurre & empleo y del empleo a los 35 años. \\
desde el primer empleo a una etapa en la que se & Segmento Primario. Ocupaciones asalariados \\
habría alcanzado cierta "madurez ocupacional". & registrados y trabajadores por cuenta propia \\
Se optó por definir los procesos de inserción a & registrados, profesionales o no profesionales con \\
partir de la movilidad entre segmentos del & capital. \\
mercado de trabajo que permitan dar cuenta de la & Segmento Secundario. Trabajadores asalariados no \\
existencia o no de procesos de estancamiento de & registrados. Trabajadores por cuenta propia sin \\
las trayectorias en posiciones del segmento & calificación y no registrados. \\
secundario. &
\end{tabular}

\begin{tabular}{|c|c|c|}
\hline \multicolumn{3}{|c|}{ Variables de estratificación } \\
\hline Variable & Concepto & Indicadores \\
\hline $\begin{array}{l}\text { Cohorte } \\
\text { de Ingreso } \\
\text { al } \\
\text { mercado } \\
\text { de trabajo }\end{array}$ & $\begin{array}{l}\text { Los componentes de las distintas cohortes compartieron los } \\
\text { mismos contextos socio-históricos, esto nos permitiría inferir } \\
\text { en qué medida el factor estructural tiene efectos sobre los } \\
\text { fenómenos a explicar. } \\
\text { Corresponde con un cambio de estrategia de desarrollo } \\
\text { económico con importantes consecuencias sociales: 1) El } \\
\text { período anterior a 1976, que se corresponde con la etapa del } \\
\text { modelo de sustitución de importaciones. 2) Desde 1976, } \\
\text { corresponde a la etapa de apertura económica. }\end{array}$ & $\begin{array}{l}\text { Año de ingreso al mercado } \\
\text { de trabajo. 1) Hasta 1976. 2) } \\
\text { Desde } 1976 .\end{array}$ \\
\hline $\begin{array}{l}\text { Origen } \\
\text { social }\end{array}$ & $\begin{array}{l}\text { Las categorías utilizadas para definir las condiciones } \\
\text { socioeconómicas del hogar de origen responden a un } \\
\text { criterio de estratificación de los sectores populares asociado } \\
\text { tanto a la calificación de la ocupación como a las } \\
\text { características del empleo del principal aportante de ingresos } \\
\text { en el hogar cuando el entrevistado tenía } 14 \text { años. Se } \\
\text { definieron dos estratos: } 1 \text { ) Estratos medios y bajos } \\
\text { integrados. 2) Estrato marginal. }\end{array}$ & $\begin{array}{l}\text { Indicadores. Categoría } \\
\text { ocupacional, grupo de } \\
\text { ocupación, registro de la } \\
\text { actividad, cantidad de } \\
\text { trabajadores en el } \\
\text { establecimiento. } \\
\text { Credenciales educativas } \\
\text { obtenidas. }\end{array}$ \\
\hline
\end{tabular}

